\title{
[ömpulter-Aidedederign
}

\section{Reusability and Flexibility in Parametric Surface-based Models: A Review of Modelling Strategies}

\author{
Aritz Aranburu ${ }^{1}$ (D), Daniel Justel ${ }^{2}$ (D) and Ivan Angulo ${ }^{3}$ (D) \\ ${ }^{1}$ Mondragon Unibertsitatea, aaranburug@mondragon.edu \\ ${ }^{2}$ Mondragon Unibertsitatea, djustel@mondragon.edu \\ 3Mondragon Unibertsitatea, ivan.angulo@alumni.mondragon.edu \\ Corresponding author: Aritz Aranburu, aaranburug@mondragon.edu
}

\begin{abstract}
CAD systems are indispensable tools in the product design and development process. Through the creation of parametric 3D models, they increase productivity, enable the design of highly complex parts, improve collaboration between different work teams and reduce time to launch. Nevertheless, during the design process there are many different modelling solutions to generate any one part, and the robustness and flexibility of a model depends to a great extent on the experience of the designer. This dependence on individuals rather than methodologies has a negative impact on downstream engineering activities, as many models are not easily editable. Developing a methodology to produce flexible and reusable 3D models is therefore key to reducing the design time of the product development process. In this paper, we review the state of the art with regard to flexibility and reusability in parametric surface-based models. We identify gaps in the field and determine the principle aspects in CAD development workflows which influence flexibility and reusability. We aim to expand the field of knowledge and establish a foundation for future research into the development of a surface-based modelling methodology.
\end{abstract}

Keywords: $C A D$, modelling methodology, surface modelling, reusability, flexibility DOI: https://doi.org/10.14733/cadaps.2021.864-874

\section{INTRODUCTION}

As companies strive to compete in a competitive global marketplace, finding ways to maximise competitive advantage is critical to success. In the design and development of products, computers have been playing an increasingly prominent role, and today, CAD systems are indispensable tools in the process of designing and developing products. Such systems increase the productivity and efficiency of the design project [19], as they enable the creation of parametric 3D models, improve collaboration between different work teams, manage the entire life-cycle of the product, and reduce time to launch.

In industrial companies, CAD systems are used to create or modify individual parts and assemblies, to develop products, and to undertake various engineering activities such as analysis, optimisation and simulations. The most extended and standardised CAD systems in the industrial sector are based on parametric associative technology [3],[4], which has a historical approach [4]. 
To increase the productivity and efficiency of any design project, a CAD efficiency strategy is critical for companies to maximise the potential of their CAD systems. Bodein et al. [2] analysed CAD systems in the automotive sector and defined five principle aspects for improvement: (i) reduce design time in all design phases (conceptual, preliminary or detailed), (ii) reuse existing CAD models and geometry, (iii) automate routine design tasks based on knowledge-based engineering (KBE) applications, (iv) enhance collaboration between designers; and ( $\mathrm{v}$ ) improve the general quality of CAD models. Therefore, to improve CAD efficiency Bodein et al. [2] proposed a roadmap with five phases: (i) standardisation, (ii) methodology, (iii) generic modelling, (iv) expert rules and (v) automation.

During part design, there are many possible modelling procedures in the solution space to generate any one part. Since creating CAD models of complex products is a "trial and error" process [1], two different designers may choose very different processes, the result of which being two different Generative Shape Design Trees (GSD trees) to generate the exact same part. However, as Amadori et al. [1] stated, one approach will be inherently more flexible than the other, due to the capacity of models to represent a wide range of different versions during re-edition [1]. Although the desired geometry is generated, not all models will be easily re-edited by another designer because the reusability of the model depends upon the procedure determined by the original designer [3]. This dependence on individuals rather than methodologies has a negative impact on downstream engineering activities, such as manufacturing, engineering analysis and optimisation. Developing robust and reusable 3D models is therefore key to reducing the design time of the product development process [6]. If the model is robust it can be easily re-edited without error propagation during tree features and subsequent model changes will not result in unpredictable and unwanted behaviour.

To achieve an efficient process during the different design and engineering phases, and to model parametrised and adaptive products during the product design phases, the strategy, modelling methods, established procedures and approaches employed are key factors. For this reason, companies often create internal design guides [4] to promote effective representation and communication of design intent between designers [5]. Part of this process involves collecting good modelling practices and reducing the possible procedures for their implementation. Thus, the need for a modelling methodology becomes clear.

Reusability in modelling methodologies is a neglected topic in the literature. Only one study was found, that of Camba et al. [4], that analyses and compares modelling methodologies of software based on associative parametric technology with a historical approach. In this study, Camba et al. [4] analysed the three solid modelling methods and identified the Resilient Modelling Strategy (RMS) [15] which generates the most reusable models; in other words, easy to edit and error-free regeneration 3D models. However, the basis of CAD systems is either classical solid-state or surface modelling [5],[23] and no studies have been identified which provide a similar comparison in the case of surface modelling. Such a comparison would greatly help CAD developers identify which modelling methodology provides the greatest flexibility and reusability for surface-based models.

According to the VDI 2209 standard [21], surface modelling is used mainly when: (i) volumeoriented modelling is not appropriate, e.g. sheet metal parts, automotive bodies etc., (ii) individual areas need to be modelled separately from the volume on the basis of their geometrical complexity, e.g. in the case of castings and forgings, and (iii) production-related aspects are paramount e.g. separate modelling of milled surfaces in mould and die-making. Given the scale of the usage of surface modelling, other industrial manufacturers would benefit from the use of reusable and flexible models to optimise the product design and development process of their products.

In this paper we review the state of the art with regards to flexibility and reusability in parametric surface-based models. We identify gaps in the field and determine the principle aspects of CAD development workflows which influence flexibility and reusability. We aim to expand the field of knowledge and establish a foundation for future research on parametric surface-based modelling. 
The reminder of the article is structured as follows. First, a relevant literature focused on reusability a flexibility of surface-based models is reviewed. Second, we discuss and propose opportunities for future research. Finally, the paper ends with the study's conclusions.

\section{RELEVANT LITERATURE}

In this section, we review the main aspects which contribute to flexibility and reusability in parametric surface-based models. First, we define reusability and flexibility and their importance in the modelling process. Second, under parametric surface modelling, we outline the common procedure of surface modelling in industry, strategic knowledge and relevant best practices. Third, we collect and analyse current history-based parametric modelling methodologies focussed on reusability in solid modelling. Finally, given that parts are not independent of their context, we analyse the design process in industry using two case studies.

\subsection{Flexibility and Reusability}

According to Amadori et al. [1] the definition of modelling flexibility is related to the robustness and design space size of the model, thus they consider those aspects:

- The flexibility of a geometrical model refers to the ability to represent a wide range of different product configurations, arrangements and sizes. The wider the range of products the model can create, the more flexible it is.

- Robustness on the other hand, refers to the errors or instability issues that changes to the geometrical model may provoke. The fewer the errors, the more robust the model is.

- The size of the design space is defined by the designer, and the range of input variables are decided by the user. For this reason it is very crucial to clearly define the input variables from the outset, as these variables affect the future behaviour of the model. If the designer is able to define a concise/limited design space, the resulting model will be robust and flexible.

The studies [1-4],[6] reviewed in this paper highlight the need for a common methodology to increase the reusability of CAD models, and thus improve collaboration between designers. Without a common methodology in place, designers can find it difficult to manage complex models in sequence, which can significantly impact workflow and productivity [3]. However, solutions to this problem a lack of attention [4] and only proposed for solid modelling.

The adaptable nature of the GSD tree allows designers to quickly model complex parts with relative ease while increasing the flexibility and reusability of their designs. Those features are organized in chronological order that is generated when a designer creates a new part. When a part is generated, three-dimensional features are added to the model in an associative relationship (parent/child) with the feature(s) they are immediately connected to, that relationship is named as dependency, Figure 1. During the design process tree-like structure is created with implicit relationship between features.

When feature dependencies are properly defined, alterations performed to a parent node will automatically propagate to its child nodes, i.e., the CAD model will react to changes in a predictable manner [4]. Therefore, identifying the optimum procedure to define dependencies is key.

Unfortunately, parent/child interdependencies between features are the root of many regeneration problems in parametric modelling. The size and complexity of a parametric CAD model can grow rapidly and significantly depending on the application. As the number of dependencies grows, so does the interconnectedness of the design tree, which may negatively affect maintainability and model reuse [4]. When feature interdependencies are not defined properly, even minor alterations may cause the CAD model to become unstable, forcing designers to rebuild the model to some degree to re-establish new design intent [4]. 


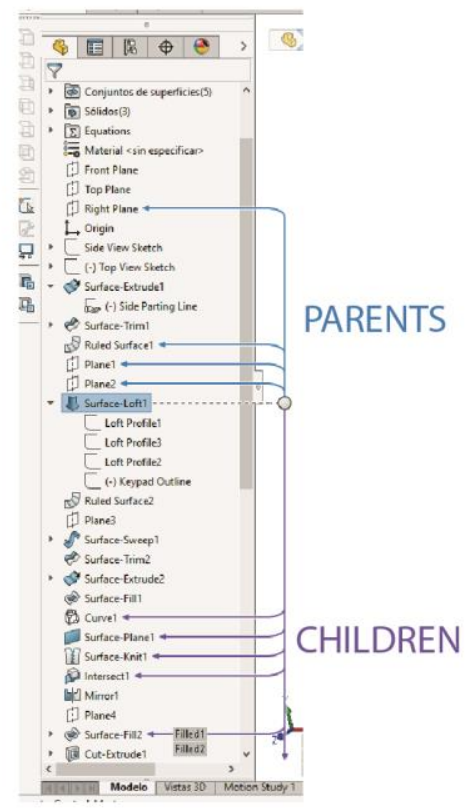

Figure 1: GSD tree and the visualization of features elements parent/child relationship in Solid Works software.

\subsection{Parametric Surface Modelling}

It is known that diverse surface modelling functions exist in CAS, NURBS-based and CAD software. Those resources are mostly used in major industries such as automotive or aeronautics. Furthermore, various approaches to KBE and workflows in the automotive CAD environment have been proposed, compared and researched in recent years [10],[11],[17],[18],[20]. However, those approaches are focused on automotive sector workflows, in multi-software conditions and having Class A surface quality as a specification of car bodies' shape or those bodies derivate parts which have the same aesthetic requirements and manufacturing related specifications.

In this research, we are focusing on parts that can be developed with CAD's common parametric surface modelling functions (e.g. sheet metal parts, castings and forging parts, moulds or plastic injected parts). Primarily, engineering-related parts. Nevertheless, aesthetics should be taken into account in some cases.

\subsubsection{Common procedure modelling}

Vukašinović and Duhovnik [22] explained the common procedure for modelling currently employed in industry. They argued that products for mass-market need diverse design tasks and require experienced designers with a great deal of work experience and knowledge of manufacturing. They took as reference the development of a surface model of a hand blender, and explain a procedure of 10 steps: (i) import the concept image, (ii) create boundary curves, (iii) generate the surface with boundary curves, (iv) free-form the generated surfaces, (v) create and delete sections on the surface, (vi) fill gaps by repeating steps ii-iv, (vii) join the surfaces, (viii) mirror and join the two surfaces, (ix) convert surfaces to solid, and ( $x$ ) add details such as fillets or chambers.

However, while this procedure guides the designer in the construction of the geometry, it does not provide sufficient criteria to decide how to start generating the geometry. Questions such as "Which parts are the most critical?" "How do we generate the surfaces to better manage the continuities or subsequent operations?" remain unanswered. In addition, as there is no specified 
criteria to generate surfaces in one manner or another, it does not consider reusability and flexibility of surface-based geometries.

\subsubsection{Knowledge needed to operate a CAD system}

To effectively operate a CAD system it is important to be fully versed in the two different types of knowledge as defined by Chester [7] and Lang et al. [13]: procedural knowledge (knowledge of the software) and strategic knowledge (knowledge needed to apply a modelling strategy). As identified by Bodein et al. [2] and Camba et al. [4], standard training methodologies are not adapted to parametric-associative CAD, and the courses that are offered by CAD vendors are based solely on procedural knowledge, i.e, understanding the purpose and limits of each function in the software. Those courses are known as "Computer Based Training" (CBT) and fail to address the strategic aspect of designing in CAD. Rynne and Gaughran [16] argued that spatial visualization, sketching and model deconstruction ability are crucial for developing efficient part modelling strategies, in other word, strategic knowledge.

While it is true that spatial visualization and sketching are skills that must be developed through time and practice, the ability to deconstruct a model is one that can benefit from the application of an established procedure. The work of Gabrielides et al. [9] and Otto and Mandorli's [14] in geometry construction has gone some way to address the strategic aspect, however to date no methodology has been found which includes resources to assist CAD users deconstruct the geometry and model according to an established and standardized procedure.

\subsubsection{Surface construction criteria}

Vukašinović and Duhovnik [22] stated that working optimally with curves and surfaces requires understanding of their mathematical properties. To achieve the desired aesthetic it is necessary to maintain the continuity of curves and surfaces during the digitalization of sketched forms. While it is clear that mathematical knowledge brings advantage to the design process, a clear and methodological way of integrating this into a modelling procedure remains unexplored in the literature.

We need criteria to know how to build a surface. These criteria must be based on, among other things, the mathematical properties of the curves and surfaces, because when modelling surfaces it is usually desirable to maintain an aesthetic quality and surface continuities that add value to the product. As Bodein et al. [3] stated, for complex solid models the selection of the feature is critical to the modelling process as it influences the parent/child references established within the model. The definition of modelling procedures for surface-based models has to integrate information that will affect the choice of the base features and the selection of references/supports during the modelling procedure. In the following section, the optimum way to construct geometries is identified.

In certain cases, we can find complex geometries that branch out, e.g. Figure 2 (a) and Figure 2 (b), in which achieving a surface with acceptable continuities is challenging. For this reason, Gabrielides et al. [9] studied how to achieve the desired continuities in various cases. They developed a process in which the desired continuities are obtained up to $\mathrm{G}_{1}$, Figure 2 (c) and Figure 2 (d). Nonetheless, this does not solve the case in which the finished quality of the surface of the product requires greater continuity than $\mathrm{G}_{1}$.

However, surface modelling has its own specifications that differentiate it from solid modelling, such as curve and surface continuities, and the aesthetic quality of the surface. Therefore it is useful to collect good practices or negative knowledge (ie, what not to do), and to analyse different surface modelling approaches. Although not performed in associative parametric software, Otto and Mandorli's [14] study of the errors in NURBS-based modelling helps to identify how to create models properly and what not to do. This study could provide an crucial inputs into the strategic knowledge base, and help designers reflect on the need for modelling methodologies and integrated guidelines. 
(a)

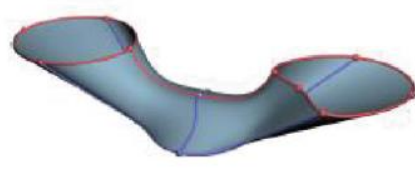

(b)

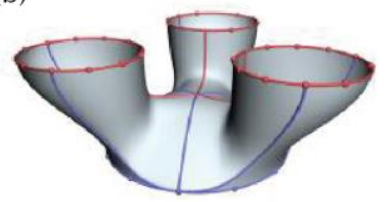

(c)

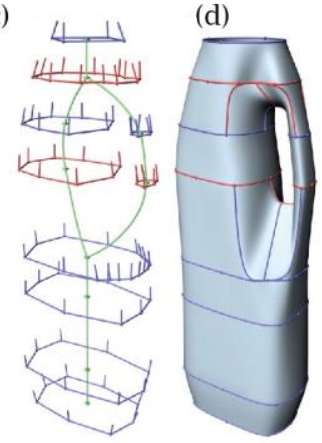

Figure 2: Branching as modelled by Gabrielides et al. [9]: (a) The final "one-to-two" surface, (b) The final "one-to-three" branching surface. (c) Contour point sets, tangent-vector estimates and the correspondence graph of the container example, and (d) The final container surface.

\subsection{History-Based Parametric Modelling Methodologies for Solid Modelling}

Although reusability and flexibility have been underworked in general [4], there are three solid modelling methodologies: [12], [3] and [15], which are compared in the research of Camba et al. [4]. The study provides an analysis of the keys elements of associative parametric technology to achieve reusability in solid models, which could be extrapolated to surface-based models

Instead, as Camba et al. [4] refers: (i) Delphi's Horizontal Modelling [12] have different problems with tree regeneration due to it is implicit horizontal strategy. Thus, it is not applicable for general use to provide reusability, and (ii) Explicit Reference Modelling [3] and Resilient Modelling Strategy [15] provides reusability for general use.

The first of two methodologies that contribute to our objective is Explicit Reference Modelling (ERM) [3]. It is focused on reducing the possible number routes to create a 3D model. The main aspects for reusability and flexibility are set out as follows [4]:

- Integrate a functional strategy to identify functional geometrical elements to obtain reusability (which is developed in more detail by Cheng and Ma [6]).

- $\quad$ Reduce interdependency features between functional geometrical parts as much as possible. Semantics simplification reduces the degrees of feature dependency and increases robustness.

- There are two categories of constraints. On the one hand, constraints to current shape are not mandatory. The priority is to use reference entities like points or planes and create sketches as simple as possible. Consequently, a positive effect of using simple features is that CAD models are easier to understand by other designers. On the other hand, there are constraints that are mandatory to the current shape. In this case the goal is to create the features as close as possible to their primitive, in order to reduce the degrees of feature dependency.

- The operations that are most susceptible to change or most volatile are placed as far down the GSD tree as possible, creating more stable and robust parts.

The second methodology is the Resilient Modelling Strategy (RMS) [15]. Camba et al. [4] demonstrated that RMS achieves greater reusability in models than ERM. The found that re-edition time for complex parts is reduced by up to $50 \%$ compared to Explicit Reference Modelling. According to Camba et al. [4], the main aspects for reusability are:

- Organizes and structures the tree in an intuitive way.

- Provides a protocol to name the elements of the tree.

- Ensures that volatile operations or those susceptible to change are as far down the tree as possible.

- Uses a skeleton as the base of the model

- Provides good practice guidelines 
In recent years, some Associative Parametric Modelling systems have started to integrate Direct Modelling Functions [4]. Direct Modelling functions are tools to quickly create and modify 3D models without any consideration of the original modelling process [4]. The benefits of these functions are that they allow direct manipulation of the model, and add flexibility to the product development workflow [4]. In addition they are easy to use, requiring a short learning curve on the part of the designer. However, they contain less options for model automation, there is no feature-to-feature associativity, and some available software packages are too immature to generate complex models [4].

\subsection{Design Process in Industry: Two Case Studies}

According to the VDI2209 standard [21], a top-down strategy can take two different approaches: on the one hand, "from the outside in" (focused on complex overall products, e.g. a complete car) and, on the other hand, "from the inside out" (oriented towards components with special requirements regarding design or production, e.g. trim parts, housing and sheet metal parts).

To gain greater insight into surface modelling, we analysed two industrial case studies that are based on a top-down strategy. The work of Xiang et al. [23] is focused on the streamlined head of high-speed trains, and the modelling of car bodies is studied by Forrai et al. [8]. It is important to note that these two examples are not modelling methodologies, but practical methods focused on the product development process workflow. We have analysed their contribution to modelling obtaining some insights.

Xiang et al. [23] explained the procedures to design the streamlined heads of high-speed trains. This work is of interest to the current review, as it describes the data collection undertaken to create a brief, as well as the many factors that need to be taken into account in such a complex project. These factors include aerodynamics, ergonomics, human factors, aesthetics, culture, and relevant standards and techniques, and require that the design team are able to work flexibly and efficiently.

The design solution space is limited in the first instance by specific sectorial standards but also by the "package". This term, which comes from the automotive sector to delimitate the maximum volume, is cleverly used as a bridge to define specification hard points (top of front window, nosecone, top of the nosecone, sectional profile, base of front window etc.) as shown on Figure 3. The package helps to ensure that design concepts are more feasible in the following step, because the CAD model is directed to future flexibility needs.

The authors [23] found that there are many constraints and hard points in this approach. The simplified sketches recall the functional approach of Cheng et al. [5], which define parametrisable characteristics embodied in CAD sketches as future modelling sources or as the skeleton resource of RMS [15]. Therefore, the use of the package is a valid resource as a reference point in the initial design process, as it helps to simplify the sketched geometry and to focus on identifying the hard points of the design.

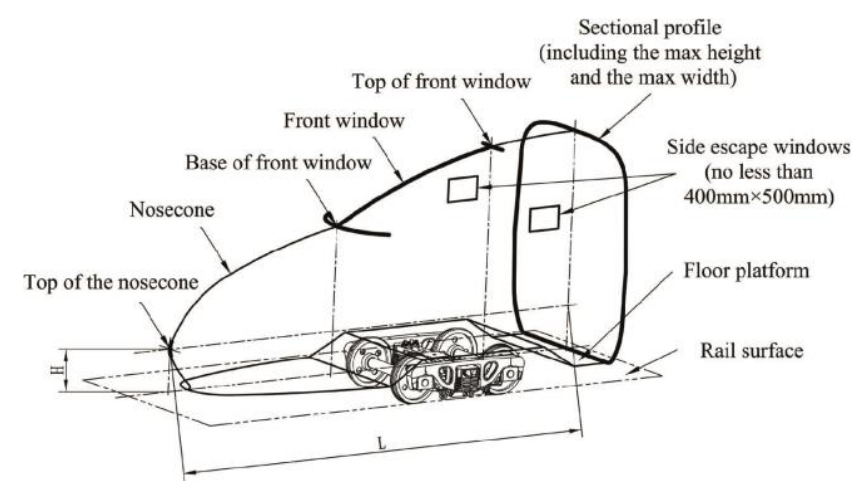

Figure 3: Package for streamlined heads of high-speed trains [23]. 
In the car bodies case study [8], the surface with the highest quality and aesthetic value which defines the product is located in a separate model file. The pieces that are generated from this first surface must be modelled in such a way that if the mother surface is restyled, the derived pieces are updated. This approach aids collaboration between designers as restyling operations can be executed in parallel with any sub-part created from mother surface. Forrai et al. [8] created intermediate pieces between the skin and the final parts, to use in calculations or as cutting elements of pieces derived from the mother skin, which are useful during downstream activities, as this approach facilitates design iterations. Forrai et al. [8] created intermediate pieces between the skin and the final parts, to use in calculations or as cutting elements of pieces derived from the mother skin, which are useful during downstream activities, because this approach facilitates design iterations.

\section{DISCUSSION AND FUTURE RESEARCH}

This review has revealed the need for research into flexibility and reusability in parametric surfacebased CAD models. While there are many options to generate a geometry solution, the robustness and reusability of the model depends greatly on the experience of the designer, Figure 4 . The main aspects to get "The Desirable Range" created by the designer's procedure, Figure 4, is what we want to state. It is clear that when a designer creates a model with a wide range of variation, they are creating a flexible model that if sufficiently robust, will streamline the design process and downstream engineering activities. However, if the design intent is not clear, and there is no simple and stable GSD tree without propagation problems, the reusability of the model by other designers will be burdensome. In such cases, the simplest solution will often be to redesign the model from scratch.

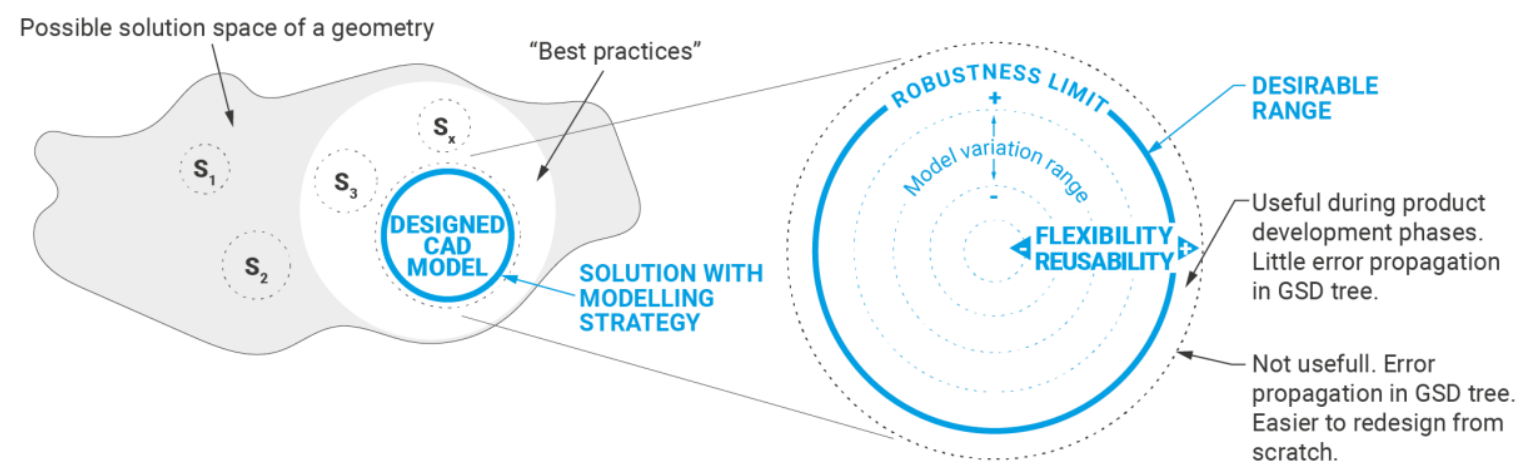

Figure 4: Solution space model identifying The Desirable Range where the optimum relationship between flexibility and reusability, and the variation range of the model is achieved. Image based on Amadori et al. [1] and Bodein et al. [3].

Against this background, we identified the opportunity to establish the next steps to further work this topic, with the end objective of defining a methodology to optimize engineering development phases. These steps are defined as the following: (i) adapt solid modelling methodologies (ERM [3] and RMS [15]) to a parametric surface modelling method, (ii) collect and select surface construction best practices and (iii) develop a geometry deconstruction strategy.

\subsubsection{ERM, RMS modelling methods and Direct Modelling}

While the advantages of using RMS to modify existing models are evident, the process proves to be too complicated when designing from scratch. This is because the heaviest workload is concentrated at the starting point, and every sketch needs to be drawn at the first stage, requiring considerable skills and experience on the part of the designer.

In surface modelling, there are more elements in the GSD tree, and hence it is necessary to determine the criteria to organise and name them. It is the opinion of this study that the strategy of 
reducing interdependency features between function parts as proposed by Bodein et al. [3] can be key to categorizing the curves and surfaces in the tree. In general, as the technology is the same, the basic principles that govern surfaces is the same. Therefore, reducing as much as possible parentchild dependencies in these new elements, will generate robust and stable models, which by default will be flexible and reusable.

Proper element organisation of GSD trees including feature categorisation according to their impact, and nomenclature based on established terminology are critical to ensure reusability and flexibility. Reducing tree dependencies and categorising surfaces and curves are areas which require further work in surface modelling and thus it would be useful to extrapolate ERM and RMS methods to surfaces. Furthermore, no cases have been found in the literature which explicitly address this issue.

Further research will focus on establishing a common systematic way to structure GSD trees, with the aim of increasing collaboration between designers and resulting in better design intent transmission.

Other possibility, we collected for increase flexibility and reusability to workflows was Direct Modelling. Nevertheless, Zou and Feng [24] proposed a methodology to work with Direct Modelling for solid parts. With this methodology, they were able to solve geometry-topology inconsistencies with robustness, demonstrating that direct modelling is a powerful resource to obtain model reusability and flexibility in workflows. However, at present, direct modelling is not powerful enough to solve complex geometries, particularly because up to G1 continuities can be lost during editing [24]. Therefore, as it is not able to be extrapolated to all cases, Direct Modelling has limited application in many industrial settings.

\subsubsection{Good practices in surface geometry construction}

In future research lines it will be necessary to incorporate best modelling practices based on continuities of curves and surfaces. This is because surface modelling has its own specifications that differentiate it from solid modelling, such as curve and surface continuities, and the aesthetic quality of the surface. These best practices will be defined after undertaking a systematic analyse different surface modelling procedures, to determine the optimum approach, as well as the negative knowledge (ie, what not to do).

\subsubsection{Geometries deconstruction strategy}

Another area that is underworked is the deconstruction of geometries [16]. The geometries in surface modelling are highly complex to visualize, and thus the establishment of clear guidelines would greatly assist in the deconstruction of the shapes. A further advantage is that this would help to develop a modelling strategy and a better organization of the GSD tree from the very initial design stages. Building on the functional approaches of Bodein et al. [3] and Cheng et al. [6], every designer can benefit from an established procedure to identify the critical properties of a part such as: functions, specification areas, and form-oriented criteria. These properties can help create the package or the skeleton during the modelling process.

In addition, according to VDI2209 [21] the usual products that are modelled on surfaces are volume oriented, sheet metal parts, casting and forging parts, separate modelling of milled surfaced in mould or die making. Therefore, any future surface-based modelling methodology should be adapted to meet the manufacturing specifications of such types products. For that reason, the product-oriented modelling strategy should always be top-down. However, depending on the needs of each product there will be two possible design approaches when modelling: from outside to inside (e.g. cars, trains, etc.) or from inside to outside (e.g. parts that require a mould for manufacture).

\section{CONCLUSION}

This study has reviewed state of the art approaches with regards flexibility and reusability in associative parametric modelling systems. Here, we have highlighted the advantages that adopting a modelling methodology can deliver, by increasing the efficiency of product design and the overall 
development process. To date however, the application of such procedures has been limited to the field of solid modelling, and no methodologies have been identified that provides reusability and reeditability of 3D surface-based models.

Given the scale of the usage of surface modelling (e.g. sheet metal parts, automotive bodies, casting parts, forging parts and mould parts), it is clear that industrial manufacturers would benefit from the use of reusable and flexible models to optimise the product design and development process of their products. This is especially relevant in cases where the potential integration of solid and surface modelling using parametric associative CAD with a historical approach is considered. Furthermore, in order to provide students with better foundations from which to approach surface modelling, clearly defined deconstruction procedures and the determination of reliable modelling best practices and guidelines will be important in the educational field. This, in turn, will generate practical knowledge centres for use of the software which will be of great benefit to this field of design.

In this study, We have identified gaps in the field of parametric surface-based modelling and have discussed the principle aspects which influence flexibility and reusability. We have, therefore, established a starting point to consolidate knowledge and thereby create a foundation for future research into the development of a surface-based modelling methodology. A methodology such as this will be critical to reducing the time expended on the product design and development process, thereby delivering cost and competitiveness benefits to industrial companies and facilitating collaboration between design partners.

Aritz Aranburu, https://orcid.org/0000-0001-6022-1153

Daniel Justel, https://orcid.org/0000-0002-8302-4862

Ivan Angulo, https://orcid.org/0000-0002-9674-5570

\section{ACKNOWLEDGEMENTS}

This work is supported by the Faculty of Engineering of Mondragon Unibertsitatea.

\section{REFERENCES}

[1] Amadori K,; Tarkian M.; Ölvader J.; Krus P.: Flexible and robust CAD models for design automation. Advanced Engineering Informatics, 26, 2012, 180-195. https://doi.org/10.1016/j.aei.2012.01.004

[2] Bodein Y.; Rose B.; Caillaud E.: A roadmap for parametric CAD efficiency in the automotiveindustry, CAD Computer Aided Design, 45(1), 2013, 198-214. https://doi.org/10.1016/j.cad.2013.05.006

[3] Bodein Y.; Rose B.; Caillaud E.: Explicit reference modeling methodology in parametric CAD system, Computers in Industry, 65, 2014, 136-47. https://doi.org/10.1016/j.compind.2013.08.004

[4] Camba J.D.; Contero M.; Company P.: Parametric CAD Modeling: An Analysis of Strategies for Design Reusability, Computer-Aided Design, 63, 2016, 18-31. https://doi.org/10.1016/j.cad.2016.01.003

[5] Cederfeldt M.; Sunnersjö S.: Solid modelling with dimensional and topological variability, International Conference on Engineering Design, Design ICED'03, Stockholm, SWEEDEN, 2003, 1-10.

[6] Cheng Z.; Ma Y.: A functional feature modeling method, Advanced Engineering Informatics, 33, 2017, 1-15. https://doi.org/10.1016/j.aei.2017.04.003

[7] Chester I.; Teaching for CAD expertise, International Journal of Technology and Design Education, 17, 2007, 23-35. https://doi.org/10.1007/s10798-006-9015-z

[8] Forrai M.; Gavačová J.; Gulan L.: A Practical Methodology for Creating Robust Parametric Surface-based Models in Automotive Engineering, Procedia CIRP, 50, 2016, 484-9. https://doi.org/10.1016/j.procir.2016.04.145 
[9] Gabrielides N.C.; Ginnis A.I.; Kaklis P.D.; Karavelas M.I.: G1-smooth branching surface construction from cross sections, CAD Computer Aided Design, 39(6), 2007, 39-51. https://doi.org/10.1016/j.cad.2007.05.004

[10] Gulanová J.; Gulan L.; Forrai M.; Hirz M.: Generative engineering design methodology used for the development of surface-based components, Computer-Aided Design \& Applications, 14(5), 2017, 642-649. http://dx.doi.org/10.1080/16864360.2016.1273581

[11] Gulanová J.; Lonek S.; Gulan L.: Comparison of two different approaches of a class-A surface creation and quality verification, Computer-Aided Design \& Applications, 15, 2018, 757-763. https://doi.org/10.1080/16864360.2018.1441242

[12] Landers D.M.; Khurana P.: Horizontally-Structured CAD/CAM Modelling for virtual concurret product and process design. US 6,775,581, 2004.

[13] Lang G.T.; Eberts R.E.; Barash M.M.; Gabel M.G.: Extracting and Using Procedural Knowledge in a CAD Task, IEEE Transactions on Engineering Management, 38(2), 1991, 57-68. https://doi.org/10.1109/17.83758

[14] Otto H.E.; Mandorli F.: Surface Model Deficiency Identification to Support Learning Outcomes Assessment in CAD Education Harald, Computer-Aided Design and Applications, 16(4), 2019, 29-51. https://doi.org/10.14733/cadaps.2019.429-451

[15] RMS. Resilient Modeling Strategy: Solids Modeling Best Practices Manual. V9 2018. https://learnrms.com. Visited on 27/02/2020.

[16] Rynne A.; Gaughran W.F.: AC 2007-2132: Cognitive modeling strategies for optimum design intent in parametric modeling (PM), American Society for Engineering Education, 2007.

[17] Salchner M.; Stadler S.; Hirz M.; Mayr J.; Ameye J.: Multi-CAD approach for knowledge-based design methods, Computer-Aided Design \& Applications, 13, 2016, 471-483. http://dx.doi.org/10.1080/16864360.2015.1131540

[18] Stadler S.; Hirz M.: A knowledge-based framework for integration of computer aided styling andcomputer aided engineering, Computer-Aided Design \& Applications, 13, 2016, 558-569. http://dx.doi.org/10.1080/16864360.2015.1131552

[19] Stroud I.; Nagy H.: Solid Modelling and CAD Systems: How to Survive a CAD System, Springer, 2011. https://doi.org/10.1007/978-0-85729-259-9

[20] Tsai Y.; You C.; Lin J.; Liu K.: Knowledge-based Engineering for Process Planning and Die Design forAutomotive Panels, Computer-Aided Design \& Applications, 7(1), 2010, 75-87. http://dx.doi.org/10.3722/cadaps.2010.75-87

[21] VDI 2209: 3D product modelling - Technical and organizational requirements Procedures, tools, and applications - Cost-effective practical use, Verein Deutscher Ingenieure, 2009:165.

[22] Vukašinović N.; Duhovnik J.: Advanced CAD modeling. Explicit, Parametric, Free-Form CAD and Re-engineering, Gewerbestrasse, Springer, 2019, 81-109. https://doi.org/10.1007/9783-030-02399-7

[23] Xiang Z.; Zhi J.; Huang J.; Kang H.; Li T.; Gao P.: A systematic approach for streamlined head form design and evaluation of Chinese high-speed train, International Journal of Rail Transportation, 2018, 1-23. https://doi.org/10.1080/23248378.2018.1501776

[24] Zou Q.; Feng H.: Push-pull direct modelling of solid CAD models, Advances in Engineering Software, 127, 2019, 59-69. https://doi.org/10.1016/j.advengsoft.2018.10.003 